\title{
Tradurre il tempo \\ Umberto Eco traduttore del cronotopo in Sylvie di Nerval
}

\author{
Susan Petrilli \\ Dipartimento di Lettere, Lingue, Arti, Italianistica e Culture comparate; \\ Università di Bari “Aldo Moro" (IT) \\ susan.petrilli@gmail.com
}

\begin{abstract}
Alla domanda “cosa vuol dire tradurre?”, Eco risponde traducendo. Infatti in Dire quasi la stessa cosa, egli si avvale soprattutto delle sue "esperienze di traduzione", espressione che fa da sottotitolo del libro. Fra queste sue esperienze particolare importanza ha la sua traduzione di Sylvie di Nerval. Il problema centrale è che cosa deve rendere il testo che traduce. Nel caso di Sylvie si tratta della "resa" del suo specifico cronotopo. Il racconto inizia con l'imperfetto e si sviluppa a partire da una perdita irreparabile, che però soltanto alla fine sarà comunicata al lettore. La traduzione deve cimentarsi con questa particolare organizzazione temporale del racconto. Questo si svolge in prima persona ed Eco ingegnosamente distingue la "voce narrante" dall'autore Gérard Nerval indicandola come Je-rard. Il "quasi” del titolo preannunzia quanto Eco dirà nelle pagine conclusive: cioè che la fedeltà nella traduzione consiste nel riconoscerla come mai definitiva. Infatti, il testo tradotto resta pur sempre lo stesso, ma altro: lo stesso altro.

To the question “what does translation mean?”, Eco responds translating. In Dire quasi la stessa cosa (Saying Almost the Same Thing) he resorts above all to his own "experiences in translation," as recites the subtitle of his book. Of his experiences particularly important is his translation of Nerval's Sylvie. The central problem is what the translating text must render. In Sylvie Eco contends with "rendering" its specific chronotope. The story begins in the imperfect recounting an irreparable loss which is only communicated to the reader at the end. Translation must experiment with this special temporal organization of the story. This is presented in first person and by naming the "narrating voice" Je-rard Eco ingeniously distinguishes it from the author Gérard Nerval. "Almost" in the title announces Eco's position formulated towards the end of his book: fidelity in translation consists in recognizing that it is never final. In fact, the translated text is always the same, but other: the same other.
\end{abstract}

\section{Parole chiave}

Eco, letteratura, cronotopo, negoziazione, testo letterario, traduzione, voce narrante,

\section{Key Words}

Eco, literature, chronotope, literary text, narrator, negotiation, translation 


\title{
Ocula ${ }^{\text {Flux_saggi }}$ \\ Occhio semiotico sui media | Semiotic eye on media
}

Susan Petrilli | Tradurre il tempo

\begin{abstract}
La comunicazione diretta esige certezza, ma la certezza è impossibile per il divenire ed è propriamente un'illusione. Se pertanto, per usare una situazione erotica, una ragazza innamorata sospirasse il giorno delle nozze, perché queste le darebbero una sicura certezza; se ella potesse stare tranquilla come donna sposata nella sicurezza giuridica; se invece di sospirare come una ragazza, ella si comportasse come una donna sposata, l'uomo giustamente dovrebbe lamentarsi della sua infedeltà, benché ella non ami qualche altro, ma perché ella avrebbe perduto l'ideale e in fondo non l'amerebbe. E questo è nel rapporto erotico l'infedeltà essenziale: quella casuale è quando si ama un altro.

Søren Kierkegaard, "Postilla conclusiva non scientifica”, 1846,

in Kierkegaard, Opere, 192.
\end{abstract}

Il libro di Umberto Eco dedicato ai problemi della traduzione, Dire quasi la stessa cosa. Esperienze di traduzione (2003) parte dalla domanda "Che cosa vuole dire tradurre?”. Si tratta di una domanda di pertinenza della scienza generale dei segni, la semiotica, ed anche della filosofia del linguaggio, dato il rapporto tra queste due discipline da Eco stesso evidenziato nel suo libro del 1984 nel cui titolo comparivano entrambe. Già nel retrocopertina del libro Dire quasi la stessa cosa, la domanda si pone in termini più "specialistici" sia per il diretto riferimento al testo - al testo letterario cui specificamente si riferiscono le sue "esperienze di traduzione" -, sia per la particolare formulazione in cui qui si presenta: "Dato il testo, che cosa di quel testo deve rendere il traduttore?". Questa "resa" non è di carattere semplicemente "tecnico" dato che essa implica inevitabilmente deliberazioni e mediazioni interpretative che hanno a che fare non semplicemente con questioni di significato, ma anche con scelte che riguardano soprattutto il senso. E quindi non è escluso, nell'incontro delle due lingue implicate, anche quello di linguaggi, di contesti, di culture, di epoche, di visioni del mondo differenti, con cui il senso ha generalmente a che fare.

Eco giustifica la presenza del "quasi" nella scelta del titolo del libro premettendo che tradurre non significa "dire la stessa cosa" in una lingua differente. La traduzione non comporta il travaso degli stessi sensi e significati da un contenitore linguistico ad un altro, da una lingua all'altra. E ciò soprattutto quando si tratta di testi letterari, quando si tratta, potremmo dire, di "traduzione di poesia", intendendo questa espressione non semplicemente in riferimento al testo poetico ma sentendola risuonare come risuona, ad esempio, l'espressione pasoliniana "cinema di poesia".

"Le esperienze di traduzione", del sottotitolo del libro, sono duplici. Esse riguardano Umberto Eco sia come autore tradotto - anche per aver spesso collaborato con i suoi traduttori -, ma anche come traduttore egli stesso. Sotto quest'ultimo aspetto, l'esperienza più importante, come egli dichiara, è la 
Susan Petrilli | Tradurre il tempo

sua traduzione in italiano del racconto Sylvie di Gérard de Nerval (1853 nella Revue des deux Mondes; 1854 in Nerval, Les Filles du feu), pubblicata nel 1999 con testo a fronte nella collana Einaudi "Scrittori traducono scrittori". A questo suo lavoro di traduzione Eco annette particolare importanza, come già aveva fatto nel libro Experiences in Translation del $2001 \mathrm{e} \mathrm{nel} \mathrm{saggio} \mathrm{dallo}$ stesso titolo, "Experiences in translation" del 1999.

Della sua esperienza di traduttore come occasione e materia delle sue riflessioni teoriche sui problemi della traduzione, Eco parla diffusamente nella "Introduzione" a Dire quasi la stessa cosa. Il riferimento oltre che a Sylvie è, sotto questo riguardo, anche a Exercices de style di Raymond Quenau - Esercizi di stile, Einaudi 1983 -, ma è soprattutto sulla traduzione di Nerval che egli ritorna nel libro. Anche in Sei Passeggiate nei boschi narrativi, Harvard University, Norton Lectures 1992-1993 (1994, XI ed. 2016), Eco ritorna più volte, proprio in riferimento a questioni di traduzione, su Sylvie ("L'ho letto a vent'anni, e da allora non ho mai cessato di rileggerlo. [...] Ogni volta che riprendo in mano Sylvie, pur riconoscendo a fondo la sua anatomia, e forse proprio per questo, me ne innamoro come se lo leggessi per la prima volta", Eco 1999b: 14-15).

Eco ricorre a Sylvie in tutti i punti salienti del percorso di Sei passeggiate nei boschi narrativi: per spiegare la differenza fra Autore, Voce Narrante o Narratore e Autore Modello (pp. 16-18, 27-30, 55); per mostrare il funzionamento del rapporto tra fabula e intreccio (46-54, 138-140); tra fabula e digressione o "indugio" (pp. 85-87); per considerare il rapporto tra mondi narrativi, finzionali, e mondo reale (pp. 101-104, 145, 155-156). In una nota al suo commento, dal titolo "La bruma, tra una parola e l'altra", alla pubblicazione Einaudi della traduzione di Sylvie (Eco in Nerval 1999: 97-139), nella nota al primo paragrafo intitolato "Perché Sylvie", sintetizzando quanto aveva già "raccontato" nel suo Sei passeggiate nei boschi narrativi, Eco elenca le diverse occasioni in cui di Sylvie si era da tempo già occupato: il saggio "Il tempo in Sylvie" (Poetica e critica, 2, 1962); una serie di seminari all'Università di Bologna negli anni Settanta; il corso alla Columbia University nel 1984; le Norton lectures alla Harvard University nel 1992-1993; due corsi a Bologna nel 1995 e alla École Normale Superieure a Parigi nel 1996. A questo racconto di Nerval è stato anche dedicato un numero speciale della rivista Versus nel 1982: Sur "Sylvie".

Per il tema che qui mi propongo di trattare è significativo che il primo lavoro dedicato da Eco al racconto di Nerval sia un saggio dal titolo "Il tempo di Sylvie". Infatti, ciò che qui mi interessa soprattutto considerare - riprendendo e sviluppando quanto ho già avuto occasione di esaminare in "Tempo di scrittura, tempo di vita nuova" (Petrilli 2012a) e anche, più recentemente (in Petrilli 2017) - è come venga presentato, nella duplice resa di Nerval e di Eco, il cronotopo letterario in cui la narrazione, come accade in altre opere letterarie, comincia dal taglio, dalla ferita, che, nel tempo reale, la morte ha prodotto.

Nella sua monografia su Nerval (1966: 178) Vito Carofiglio fa notare, riferendosi alle "Notes manuscrites" per Aurelia (I: 421), quanto sia indicativo che Nerval parli della sua ripresa nella scrittura in termini di "vita nuova" e, evocando per due volte, nel giro di poche righe, il nome di Dante, scriva: "Cet- 


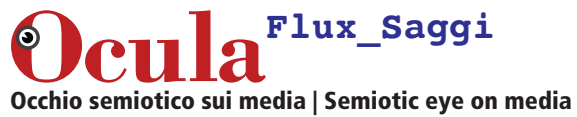

Susan Petrilli | Tradurre il tempo

te Vita nuova a eu pour moi deux phases". Nel suddetto mio saggio del 2012 i testi di riferimento, sotto tale riguardo, sono, oltre la Vita Nova di Dante, la poesia di Puškin del 1830, "Razluka" ("Separazione" o "Dipartita"), la Recherche di Proust, e di Roland Barthes: Journal de deuil (1977), tradotto in italiano con il titolo ben scelto, Là dove lei non è (2010), Vita nova (1979), La chambre claire (1980), e La préparation du roman, corso al Collège di France negli anni 1978-1980 (2003).

Dalla resa traduttiva del cronotopo specifico del racconto di Nerval ne va l'intera resa della sua "voce narrante". Eco ingegnosamente, in "Rilettura di Sylvie", il commento della sua traduzione di Sylvie (pp. 95-97), indica la voce narrante, l'“io", che racconta, per distinguerla da quella dell'autore Gérard, come Je-rard.

Je sortais d'un théâtre où tous les soirs je paraissais aux avant-scènes en grande tenue de soupirant. Quelquefois tout était plein, quelquefois tout était vide. [...] Indifférent au spectacle de la salle, celui du théâtre ne m'arrêtait guère - excepté lorsqu'à la seconde ou à la troisième scène d'un maussade chef-d'œuvre d'alors, une apparition bien comme illuminant l'espace vide, rendant la vie d'un souffle et d'un mot à ces vaine figures qui m'entouraient. Je me sentais vivre en elle, et elle vivait pour moi seul (Nerval 1999: 2).

Uscivo da un teatro, dove ogni sera mi esibivo al palco di proscenio in gran tenuta di primo amoroso. Talora tutto era pieno, talora tutto era vuoto. [...] Indifferente allo spettacolo della sala, neppure m'attirava quello del palcoscenico, - tranne quando, alla seconda o terza scena di uno di quegli uggiosi capolavori dell'epoca, un'apparizione ben nota illuminava lo spazio vuoto, ridando vita in un soffio e con una sola parola a quelle vane parvenze che mi attorniavano. Io mi sentivo vivere in lei, ella viveva per me solo (Nerval 1999: 3).

Il tempo successivo a "Uscivo da un teatro" - la successione di eventi che uno si aspetterebbe di seguito - non viene raccontato. Nessun "quando, a un tratto" come ci si aspetterebbe da un tradizionale, o banale, romanzo di avventure. Invece ciò che è raccontato prosegue da "ogni sera mi esibivo al palco del proscenio...", in cui l'informazione circa il motivo del suo recarsi ogni sera al teatro, dà occasione al ricordare, alla narrazione concernente tempi trascorsi, ricordi di un passato, che come tale, come passato soggetto al ricordare, risulta piuttosto "nebbioso".

L'inizio con l'imperfetto è abbastanza indicativo del tempo e ritmo del racconto, del suo andamento, in quanto questo tempo non specifica esattamente quando e per quanto: è il tempo che si presta a sfumare i confini temporali, è il tempo della vaghezza. Da qui il suo fascino nella narrazione letteraria. Ma soprattutto qui, in Sylvie, il tempo della narrazione è un tempo imperfetto.

Tradurre il ritmo, e il senso che nel ritmo si realizza, un senso che sfugge alla presa del "detto", per evocare Emmanuel Levinas (1974), è un tema trattato da Eco in Experiences in Translation proprio attraverso la traduzione di Sylvie. Qui egli racconta che, nonostante le sue ripetute letture di Sylvie, soltanto nel tradurre si rese conto di un mezzo stilistico impiegato di frequente da Nerval (Eco 2001: 40). 


\section{Ocula \\ Occhio semiotico sui media | Semiotic eye on media}

Susan Petrilli | Tradurre il tempo

Nella poesia propriamente detta la possibilità di realizzare il ritmo testuale è in gran parte affidata alla metrica e i suoi stilemi. Ma il ritmo scandisce anche il testo narrativo e, in certe scene, Nerval non manca di inserire nella sua prosa versi metrici (egli ricorre all'uso, ad esempio, di alessandrini, emistichi, endecasillabi) con l'effetto di conferirvi una potente qualità onirica. Eco se ne accorge traducendo. L'uso della metrica contribuisce a creare un'atmosfera irreale, accrescendo con ciò l'effetto ottenuto mediante il ricorso al tempo imperfetto - il tempo della vaghezza, proprio della scrittura letteraria - e anche alla metafora della nebbia riferita al rimembrare, alla narrazione di ricordi dal passato, confusi, indefiniti e fortemente suggestivi. L'effetto dell'imperfetto, insieme al ritmo della metrica, si traduce in corporeità e seduzione, amplifica la partecipazione in termini di intercorporeità, di immediato coinvolgimento, di fascinazione. La struttura del ritmo accentua, enfatizza, la struttura temporo-spaziale della narrazione, il suo cronotopo specifico, che, in questo caso, riesce a esaltare la relazione tra passato, presente e futuro nella loro assenza di confini precisi, nel loro reciproco sconfinamento, e a raffigurare le rimembranze di un passato che pervade il presente e si proietta altrove; e, in tutto questo, ottenendo la percezione, vaga, della relazione, sfumata, tra realtà e immaginazione. Una valida resa traduttiva deve poter ottenere, nella lingua impiegata, quasi lo stesso effetto, dare luogo quasi allo stesso senso, quasi allo stesso ritmo; e nella traduzione di Eco di Sylvie questo accade, e si potrebbe dire: anche senza quasi.

Nelle "Note sulla traduzione", che costituisce la seconda parte del testo di Eco che segue al racconto (pp. 140-165), Eco si sofferma su quanto, a un certo punto, dice "Je-rard" - nome con cui egli indica l'io, la voce, narrante, che è anche il soggetto che all'inizio usciva dal teatro (ivi: 110) - : "sans tenir compte de l'ordre des temps", espressione che segue all'indicazione di dubitare di tutte le donne, espressione tradotta in genere liberamente in italiano con "senza tener conto dell'epoca diversa", "senza eccezione alcuna di tutte le donne di ogni tempo", "senza tener conto della mutazione dei tempi".

Eco traduce questa espressione, che significativamente si trova verso l'inizio e verso la fine della narrazione, alla lettera - e cioè "senza tener conto dell'ordine dei tempi" - per quello che effettivamente significa, e come chiave di lettura del racconto di Jerard: perché "quello che in Sylvie entra in crisi è proprio l'ordine dei tempi" (ivi: 146).

Quale "ordine dei tempi"? Evidentemente quello di una narrazione storica, biografica, di una narrazione appartenente ai generi semplici, ai generi della parola diretta, oggettiva, distinti dai generi complessi, quelli della parola indiretta, oggettivata, raffigurata, cioè dai generi letterari (v. Bakhtin 1979, tr. it.: 245-290). Ma è messo in crisi anche l'ordine dei tempi di altri generi narrativi, come per esempio il romanzo poliziesco. In Sylvie avviene il contrario di ciò che avviene in un racconto poliziesco, in cui ci sono sparsi indizi che portano alla scoperta della verità. Qui invece il racconto "depista", vuole appunto "farci perdere l'ordine dei tempi” (Eco in Nerval 1999: 130).

Il tempo, in Sylvie, tende a confondersi: Eco (ivi: 131) cita Shakespeare: time is out of joint. Questa "confusione", per quanto riguarda il tempo nella narrazione, è collegata con vuoti di memoria sia di Jerard sia degli altri perso- 


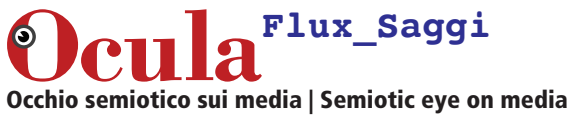

Susan Petrilli | Tradurre il tempo

naggi, della stessa Sylvie che solo a un certo punto, alla fine del testo, si ricorda che Adrienne è morta.

Intanto per quanto riguarda lo stesso narratore, Jerard, non è mai possibile sapere, dal racconto, con quale dei suoi io si identifichi, al punto che, come dice Eco, il pronome "Io" designa sempre un Altro (ivi: 110-111). L’identità di Jerard resta confusa, e se nella storia una volta egli si nomina, lo fa indicandosi come "un ignoto". Inoltre, talvolta sotto Jerard compare l'autore, Nerval, ma generalmente in una maniera tale da lasciare ambigua l'identificazione. Il teatro menzionato all'inizio della narrazione ritorna per tutto il discorso, come esplicitamente indicato nei travestimenti dei personaggi e come allusione dell'avvicendarsi in tutta l'opera, di teatro e realtà (cfr. ivi: 114).

Incerti sono i tempi del racconto e incerti sono anche gli spazi. In ogni spostamento, in ogni viaggio Jerard sembra girare in tondo come "falena impazzita"; e questa struttura temporale può essere vista come una "metafora temporale": "non è tanto Jerard che gira nello spazio, è il tempo, il suo passato che danza in cerchio intorno a lui" (Eco, ivi: 125).

Alla fine del racconto, come abbiamo accennato, Sylvie rivela a Jerard che Adrienne è morta molto tempo prima. Più esattamente è Jerard che si ricorda di dire al lettore che Adrienne è morta. Nel racconto, infatti, la notizia è introdotta dalla "voce narrante", da Jerard, con un semplice "dimenticavo di dire" (Nerval 1999: 89). Ciò che ha dimenticato di dire è che, avendo chiesto, per caso, a Sylvie, "Vi ricordate di Adrienne?" a proposito di una certa somiglianza tra quest'ultima e l'attrice dello spettacolo che avevano visto insieme (lo spettacolo era dato dalla compagnia di cui faceva parte Aurélie):

Ella scoppiò a ridere dicendo: "Che idea!" Poi, come pentita, con un sospiro aggiunse: "Povera Adrienne! È morta nel convento di Saint-S***, verso il 1832" (ibid.)

Elle partit d'un grand éclat de rire en disant: "Quelle idée”. Puis comme se le reprochant, elle reprit en soupirant: "Pauvre Adrienne! Elle est morte au convent de saint-S***, vers 1832 ".

E così termina Sylvie.

La data finale non è "un rintocco funebre che chiude la storia" (Eco in Nerval 1999: 138), ma ne è invece il momento di avvio, l'avvio della ricerca di un tempo perduto. Questa data non è ciò che segna un "fallimento di un'impresa disperata", ma ciò che produce l'opera riuscita di Sylvie. Nessuna "sconfitta" di Nerval, e nessuna "vittoria sul Tempo" da parte di Proust, perché come lo stesso Eco dice:

Dunque non è che Jerard (o con lui Nerval) smetta di narrare quando capisce che tutto è finito: al contrario, è proprio dopo che ha capito che tutto è finito che incomincia a narrare (e a narrare di un se stesso che non sapeva né poteva sapere che tutto era ormai finito) (ivi: 139).

Né in ciò che la scrittura restituisce è "azzerato il passato"; esso non è né qualcosa "per cui vale la pena di vivere", né qualcosa "per cui vale almeno la pena di morire". La scrittura si limita a mostrare la possibilità di una vita nova 


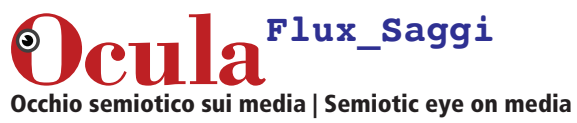

Susan Petrilli | Tradurre il tempo

nei rapporti con gli altri, con le cose, con il tempo, con il mondo, con se stessi. In maniera rinnovata, rinnovata in Proust rispetto a Nerval, ma senza velleità di "superamento", di competizione, di "sfida" (v. ibid.)

La notizia che Adrienne è morta avviene nel racconto di Jerard, il quale perciò sapeva fin dall'inizio. Sicché anche qui è dallo strappo, dall'interruzione, dalla sofferenza, che la morte ha prodotto, l'inizio della narrazione, l'inizio della scrittura, il suo modo di procedere, di ricostruire, di ricordare, di ritrovare il tempo perduto. Anche qui dunque la morte, la consapevolezza che "tutto è finito", non ostacola la narrazione, ma, al contrario, la provoca, la motiva, la orienta, le conferisce il tono, il motivo, il ritmo. A partire dalla perdita non solo ha inizio la ricerca di un tempo perduto, ma ha inizio anche un tempo ritrovato, prende avvio la "preparazione del romanzo" intesa anche come costruzione di una "vita nova" (v. Barthes, La préparation du roman, 2003).

L'altro tempo della scrittura letteraria è anche un altro spazio, un altro luogo, fuori dai luoghi comuni della realtà. Da quest'altro tempo e da quest'altro spazio, il rapporto realtà-follia è rovesciato, e con Maurice Blanchot si può parlare della "realtà" come "follia del giorno". Come osserva Vito Carofiglio nel libro Nerval e il mito della "puretée”, 1966: 170):

la dialettica tra razionale e irrazionale, logico e onirico, realistico e puramente fantastico, non si risolve in Nerval con la vittoria dell'uno sull'altro, ma con una specie di accordo instabile (drammatico) tra l'uno e l'altro.

Riferendosi a Gérard de Nerval, e in particolare a Sylvie, Proust, in Contro Sainte-Beuve (1974: 30), osserva che la "follia" della scrittura letteraria rispetto alla realtà può consistere nell'attribuire più importanza a un sogno, a un ricordo, alla singolarità di una sensazione, rispetto a "ciò che è comune a tutti, percepibile a tutti, la realtà":

Chez Gérard de Nerval la folie naissante et pas encore déclarée n'est qu'une sorte de subjectivisme excessif, d'importance plus grande pour ainsi dire, attachée à un rêve, à un souvenir, à la qualité personnelle de la sensation, qu'à ce que cette sensation signifie de commun à tous, de perceptible pour tous, la réalité.

In questa disposizione, che è in fondo la disposizione artistica, la realtà è considerata, dice Proust citando Flaubert, solo per "l'emploi d'une illusion à décrire", "l'impiego di un'illusione da descrivere", trasformando in una sorta di realtà le "illusioni" considerate degne di essere descritte.

Gli esseri che incontriamo, osserva Proust, riferendosi a Sylvie, ma forse anche alla "realtà" non realisticamente intesa, sono come la donna dei versi con cui termina Fantasie (in Nerval, Petits châteaux de Bohême, 1853), "una femme / Que dans un autre existence peut-être / J'ai vue et dont je me souvienne", una donna che Gérard (con Eco, dovremmo scrivere Je-rard) aveva conosciuto in un'altra esistenza e di cui serbava il ricordo (v. ivi: 34). E sempre parlando di Sylvie, ma come se stesse parlando degli effetti che le illusioni e allusioni della scrittura letteraria producono sul lettore, osserva: 


\section{Ocula ${ }^{\text {Flux__aggi }}$ \\ Occhio semiotico sui media | Semiotic eye on media}

Susan Petrilli | Tradurre il tempo

Mais on ne nous rend pas le trouble que nous donne notre maîtresse en parlant de l'amour, mais en disant ces petites choses qui peuvent l'évoquer, le coin de sa robe, son prénom.

Il turbamento che suscitava in noi la nostra amante non ci viene ridato da chi parli dell'amore, ma da chi ricordi le piccole cose che possano rievocare la presenza di lei: la stoffa del suo vestito, il suo nome... (ivi: 37 ).

E conclude, ancora a proposito di Sylvie:

Mais tout compte fait, il n'y a que l'inexprimable, que ce qu'on croyait ne pas réussir à faire entrer dans un livre qui y reste. C'est quelque chose de vague et d'obsédant comme le souvenir. C'est une atmosphère. L'atmosphère bleuâtre et pourprée de Sylvie. Cet inexprimable-là, quand nous ne l'avons pas ressenti nous nous flattons que notre oeuvre vaudra celle de ceux qui l'ont ressenti, puisqu'en somme les mots sont les mêmes. Seulement ce n'est pas dans les mots, ce n'est pas exprimé, c'est tout entre les mots, comme la brume d'un matin de Chantilly.

Ma, tutto sommato, solo l'inesprimibile, solo quello che si credeva di non poter far entrare in un libro, resta in questo. Qualcosa d'indefinito e di ossessionante come il ricordo. Un'atmosfera. L'atmosfera bluastra e purpurea di Sylvie. Questo inesprimibile, quando non lo abbiamo sentito, ci illudiamo che la nostra opera possa esprimerlo quanto quella di chi lo abbia sentito, perché, in definitiva, le parole sono le medesime. Ma esso non sta nelle parole, non è espresso: sta tutto tra una parola e un'altra, come la nebbia di un mattino di Chantilly (ivi: 38 ).

Il tempo del "cronotopo" letterario è collegato con luoghi, città, nomi geografici e nomi di persona, eventi storici, ricorrenze, come la festa, situazioni, come il ballo, con particolari momenti del giorno, l'alba, il meriggio, il tramonto, "la sera del dì di festa" (Leopardi), con il tempo in senso meteorologico, "il tempo che fa".

Sotto quest'ultimo aspetto un riferimento non trascurabile può essere quello a Aziyadé di Pierre Loti. Questo autore ricorre in Contre Saint-Beuve di Proust, e ci conduce a Roland Barthes, alla sua "Presentazione" di Aziyadé (tr. it. 1971). Barthes (ivi: 16) descrive Aziyadé come un libro fatto di eventi (incidents), dove "l'incident, déjà beaucoup moins fort que l'accident (mais peut-être plus inquietant) est simplement ce qui tombe doucement, comme une feuille, sur le tapis de la vie", "l'evento (incident), già meno forte dell'incidente (accident), ma forse più inquietante, è semplicemente quel che cade con dolcezza, come una foglia, sul tappeto della vita [...]”. Una foglia che cade con dolcezza sul tappeto della vita: non possiamo fare a meno di andare con la memoria alla scena iniziale del film Forrest Gump di Robert Zemeckis.

Scrive Proust:

Ainsi [...] ce sont les mots Chââlis, Pontarmé, îles de l'île-de-France, qui exaltent jusqu'à l'ivresse la pensée que nous pouvons par un beau matin d'hiver partir voir ces pays de rêve où se promena Gérard. 


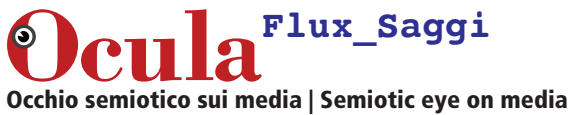

Susan Petrilli | Tradurre il tempo

Così, sono i nomi di Chââlis, di Pontarmé, di isole dell'Île de-France, a esaltare in noi sino all'ebbrezza il pensiero che, un bel mattino d'inverno, potremmo benissimo recarci in quei paesi di sogno nei quali passeggiò Gérard de Nerval (Proust 1974: 37).

Nel testo intitolato "Rilettura di Sylvie" che segue alla sua traduzione con testo a fronte pubblicata nella collana Einaudi "Scrittori traducono scrittori" (Nerval 1999), Eco tratta dell'uso dell'imperfetto indicativo, "il tempo imperfetto" - che come abbiamo visto ha un ruolo importantissimo in Sylvie perché "è il tempo che ci deve fare perdere i confini del tempo" (Eco 1999: 126) - nella prima parte, dal titolo "La bruma, tra una parola e l'altra". Non se ne occupa più poi nella seconda parte, "Note sulla traduzione", perché in italiano la traduzione dal francese dell'imperfetto non costituisce un problema.

Se ne occupa invece nelle sue Norton Lectures (1992-1993), alla Harvard University (tr. it. Sei passeggiate nei boschi narrativi, 1994: 15-16) avendo a che fare con la lingua inglese, che non ha l'imperfetto. Eco cita le soluzioni diverse date nella edizione del 1887 e in una del 1932 circa la traduzione dell'imperfetto dell'inizio di Sylvie ("Je sortais d'un théâtre où tous le soirs je paraissais aux avant-scènes en grande tenue de soupirant”):

I quitted a theater where I used to appear every night in the proscenium...

I came out of a theater where I appeared every night...

In una lingua, come l'inglese, in cui non c'è l'imperfetto, bisogna rendere in qualche modo il suo duplice carattere durativo (indica un'azione che dura, che non è compiuta) e iterativo (indica un'azione che si ripete). In molti casi, come in particolare in Sylvie, è al tempo stesso entrambe le cose. Il "sortais" dell'inizio di Sylvie, osserva Eco, è durativo, ma "paraissais" è sia durativo, sia ripetitivo:

A causa di questa sua ambiguità temporale l'imperfetto è il tempo in cui si raccontano i sogni, o gli incubi: ed è il tempo delle fiabe. "Once upon a time" in italiano si dice "c'era una volta”, "una volta” può essere tradotto con "once”; ma è l'imperfetto c'era che suggerisce un tempo impreciso, forse ciclico, che l'inglese rende con "once upon a time" (ivi: 16).

L'inglese può esprimere la iteratività del "paraissais" francese o accontentandosi dell'indicazione testuale "every evening". Non si tratta di un incidente da poco perché il gran fascino di Sylvie si basa su una calcolata alternanza di imperfetti e passati remoti, e l'uso intenso dell'imperfetto conferisce a tutta la vicenda un tono onirico, come se stessimo guardando qualcosa con gli occhi semichiusi. Il lettore modello a cui pensava Nerval non era anglofono, perché la lingua inglese era troppo precisa per i suoi scopi (ibid).

Un altro inconveniente dovuto al passaggio dalla lingua francese alla lingua inglese come pure alla lingua italiana riguarda la proposta di Eco di indicare la voce narrante con "Je-rard". In "Rilettura di Sylvie", testo aggiunto da Eco alla fine della sua traduzione di Sylvie (1999), egli tuttavia adotta in italiano 
Susan Petrilli | Tradurre il tempo

questa convenzione (ivi: 110). Invece, in Sei passeggiate nei boschi narrativi (1994), in quanto corrispondente al testo inglese delle "Norton Lectures", Eco scrive così: "Con i miei studenti avevamo deciso di chiamarlo ["colui che dice 'io' nel racconto"] Je-rard, ma siccome il gioco di parole è possibile solo in francese lo chiameremo il Narratore" (ivi: 17).

Per quanto riguarda il rapporto tra francese e italiano nella traduzione di Sylvie, per cui si deve alcune volte rinunciare alla traduzione letterale ovvero ad "essere lessicalmente fedeli", menziono qui due casi segnalati da Eco in "Note sulla traduzione" (Nerval 1999: 140-165): quello della traduzione del titolo del capitolo 14, "Dernier feuillet", l'ultimo, di Sylvie, che si conclude con l'indicazione di un anno, il 1832, in cui Adrienne "est morte" (Eco fa riferimento alla traduzione di questo titolo anche in Dire quasi la stessa cosa, 2003: 97-98); e quello della traduzione di fauvettes, che Jerard aveva detto che Sylvie aveva in una gabbia, ma quando poi visita la camera di Sylvie rileva che non c'erano les fauvettes, ma des canaris (v. Eco, "Note sulla traduzione", in Nerval 1999: 140-142).

Nel secondo caso, alla traduzione fedele si deve rinunciare perché in italiano le fauvettes si chiamano "silvie": le silvie di Sylvie! Eco sceglie di tradurre con "capinere", "anche perché capinera è parola molto dolce" (ivi: 142).

Nel primo caso, quello del titolo del capitolo finale, "Dernier feuillet”, l'unico in cui è data l'indicazione di una data, sia pure in forma vaga, imprecisa, "verso il 1832", presunto anno della morte di Adrienne - che viene annunciata al lettore dalla voce narrante, da Jerard, introducendo la notizia (l'abbiamo visto) con un "dimenticavo di dire" -, feuillet è ciò che i bibliofili (Nerval lo era) chiamano "carta". Ma "Ultima carta" ha in italiano una connotazione che è assente in francese. Dunque la scelta di Eco "Ultimo foglio".

Abbiamo già detto che nel racconto tempi e spazi si confondono in maniera inestricabile. Eco (in Sei passeggiate nei boschi narrativi, 1994: 29), a questo proposito, si sofferma sul “j”y" e sulla sua possibilità di traduzione (è un "là" o un "qui"?) della frase "Pendant que la voiture monte les côtes, recomposons les souvenirs du temps où j'y venais souvent” (Nerval 1999: 22). E si chiede anche a chi attribuire questa frase: al narratore (a "Jerard") o, come invece risulta più opportuno, all'Autore modello (che Eco propone di indicare come "Nerval")?

Nella p. 64 di Dire quasi la stessa cosa, Eco parla dell'uso abbondante, in Les Filles $d u \mathrm{Feu}$, di virgolette e di trattini, quest'ultimi usati non solo per il discorso diretto, altrui, ma anche per il discorso indiretto libero, per incisi, per osservazioni parentetiche. Ciò, dice Eco, contribuisce a confondere le voci, gli eventi, fondendo accadimenti nel presente e ricordi, facendo perdere il distanziamento temporale e spaziale (v. anche pp. 142-143 in Nerval 1999). Eco aggiunge una nota dove osserva che "Sylvie è caratterizzato dal fatto che solo apparentemente la narrazione è in prima persona"; spesso interviene la voce dell'Autore "a commento di quello che il personaggio-narratore dice". Il risultato è l'andamento dialogico che la narrazione va assumendo.

Così, nella frase sopra citata - "Pendant que la voiture monte les côtes, recomposons les souvenirs du temps où j'y venais souvent", "E mentre la carrozza sale lungo i pendii, ricomponiamo i ricordi del tempo in cui ci venivo tanto 
Susan Petrilli | Tradurre il tempo

spesso" -, malgrado il suo apparente carattere monologico, Eco individua un dialogo tra tre persone: "Nerval", ovvero l'Autore modello che si inserisce surrettiziamente nel discorso del narratore, "Jerard" il narratore-eroe, perché è lui che in quei luoghi "venais souvent", e noi, i lettori, chiamati in causa altrettanto surrettiziamente, e coinvolti nella ricostruzione dei ricordi.

Sono appunto queste le dramatis personae - l'Autore modello, identificabile nello Stile e nella Forma del testo, il Narratore, l'Eroe (coincidente o meno con il Narratore), il Lettore modello -, ciascuna con il proprio tempo, il proprio cronotopo, le proprie relazioni reali e immaginarie, di cui il Traduttore deve tener conto, calcolando puntualmente gli effetti che la trasposizione da una lingua all'altra comporta sul dialogo che l'Autore modello ha stabilito tra di esse.

Nel caso specifico della scrittura letteraria la traduzione comporta, in qualche modo, per usare l'espressione impiegata Antonin Artaud (1961, 1989) a proposito della sua traduzione di Alice Though the Looking Glass di Lewis Carroll, una sorta di "impresa antigrammaticale". Si tratta di spingere la propria lingua, la lingua in cui si traduce, fino all'incontro con una lingua altra, ma non semplicemente tra loro, come può accadere in un libro di grammatica di una lingua straniera $\mathrm{o}$ in un dizionario bilingue, ma attraverso la complicità del testo, del testo letterario, del testo di scrittura. Nella traduzione da parte di Umberto Eco, il testo Sylvie di Nerval risulta un ottimo "Galeotto" per l'incontro tra la lingua francese e la lingua italiana, e attraverso di esso si riesce effettivamente a mostrare che cosa significa riuscire a dire quasi la stessa cosa traducendo, in cosa consista la "negoziazione" del tradurre, la "fedeltà" della traduzione.

La scrittura letteraria guarda le cose con lo sguardo indiretto, con la "coda dell'occhio" (Poe 1987; Ponzio 2016), secondo una prospettiva che la traduzione del testo amplifica e sviluppa. Il passaggio da una lingua all'altra contribuisce alla possibilità di uscire dai limiti del mondo con cui una lingua qualsiasi tende a coincidere, di uscire dall'ordine del discorso, dalla rappresentazione, dall'ontologia, dall'essere-così delle cose (cfr. Ponzio 2007, n. ed. 2018). Analogamente, secondo una prospettiva "extralocalizzata", lo sguardo indiretto, differito della traduzione, il suo "discorso indiretto mascherato da discorso diretto", come dice Eco riferendosi anche a un mio saggio in cui ciò è particolarmente evidenziato (v. Petrilli, ed. 1999: 11-17; 2014b: 104-110), permette di scorgere ciò che sfugge allo sguardo diretto e anche di intravedere, rispetto all'“ordine del discorso" (Foucault 1970), rispetto ai luoghi comuni del discorso, la possibilità del "fuori luogo", di scorgere "l'esorbitante nella produzione dell'identico" (Ponzio 2013), assumendo anche la valenza di una "sovversione non sospetta" (Jabès 1982). Come lo scrittore, anche il traduttore si ritrova nella condizione di dover "lottare" con la propria lingua, sentendone tutta la materialità semiotica, l'oggettività segnica, l'estraneità. Lo scrittore-traduttore è colui che sperimenta direttamente il potere della lingua - il linguaggio verbale come legislazione, la lingua come codice -, è colui che sa che la lingua più che permettere di dire obbliga a dire (Barthes 1978) in questo senso la lingua è tiranna.

Ma grazie ad espedienti come quelli offerti dalla traduzione e dalla scrittura letteraria, pur non potendo uscire dai limiti del linguaggio (Wittgenstein, 
Susan Petrilli | Tradurre il tempo

Tractatus, 1922), è possibile uscire dalla presa della propria lingua, sottrarsi all'obbligo di un atteggiamento strumentale e, al tempo stesso, servile rispetto al potere che esercita e aprire alla critica nei confronti di legislazioni e codici che l'ordine del discorso impone.

Ma l'exotopia dello sguardo indiretto dello scrittore e del traduttore favorisce anche, paradossalmente proprio tramite il distanziamento che lo caratterizza, la prossimità, il sentire non-indifferente, tra l'io e l'altro, tra testi e contesti diversi e lontani; permette la partecipazione alla vita nella forma di un amore per essa che è anche innamoramento di ciò che è lontano, amore che avvicina e umanizza, che esorbita dalla responsabilità "tecnica", di ruolo, e impegna, invece, in una responsabilità illimitata e partecipe nei confronti dell'altro, del prossimo lontano e anche del lontano ormai sempre più prossimo (v. Ponzio e Petrilli 2016: 35-36).

Il lavoro di Eco sulla traduzione va senz'altro nella direzione della valorizzazione del compito del traduttore (v. Ponzio 2017), a cui si richiede tutt'altro che il nascondersi dietro la maschera dell'invisibilità, dell'indifferenza, dell'anonimato secondo il presupposto tacito di un mito, quello della "oggettività" della traduzione. E qui va precisato che, contrariamente a ciò che si intende per "oggettività" con riferimento alla "materialità semiotica", all'alterità di significazione accennata sopra, cui consegue il carattere aperto del percorso interpretativo, qui invece l'"oggettività" riguarda la pretesa possibilità di qualcosa di fisso e definitivo che faccia da garanzia al "significato monologico", cui tanto più si perviene quanto meno si abbia "voce in capitolo", in palese contrasto con il costitutivo carattere dialogico dei segni e dei testi, a maggior ragione quando tale dialogo intercorre nello spostamento tra lingue diverse.

Di conseguenza, attraverso la riflessione di Eco sulla traduzione, inscindibile dalla sua dedizione di traduttore, si intuisce il legame profondo, omologico, tra traduzione e scrittura letteraria, e anche tra traduzione e discorso amoroso (Barthes 2007; Ponzio, "Introduzione", in Barthes 2007), in quanto si tratta pur sempre in essi di sperimentazioni spazio-temporali, cronotopiche dove le voci si incontrano e si confondono nella ricerca dell'unicità, dell'irripetibilità, dell'alterità del proprio "oggetto amato". Eco traduttore di Sylvie direttamente o indirettamente (1999b: 14-15) se ne dichiara innamorato, e racconta del suo ritornare sempre di nuovo a questo testo, con lo stesso trasporto della prima volta, impegnato a s-velarne e ri-velarne le risonanze singolari.

Eco non perde occasione di potersi avvalere, occupandosi della questione del tradurre, della possibilità di ritornare sul mito della "lingua perfetta" (v. Eco 1993a), sulla ricerca e sulla costruzione di una lingua unica da sostituire alla "babele" della molteplicità delle lingue. E, con la sua consueta sottile capacità argomentativa, osserva:

Il limite di una lingua universale veicolare è lo stesso delle lingue naturali su cui viene ricalcata: essa presuppone un principio di traducibilità. Se una lingua universale veicolare prevede di poter rendere i testi di qualsiasi lingua è perché, malgrado esista un "genio" delle singole lingue, benché ogni lingua costituisca un mondo assai rigido di vedere, organizzare e interpretare il mondo, si assume che sia pur sempre possibile tradurre da lingua a lingua (Umberto Eco, La lingua perfetta, 1993a, p. 371). 


\section{Ocula ${ }^{\text {Flux_saggi }}$ \\ Occhio semiotico sui media | Semiotic eye on media}

Susan Petrilli | Tradurre il tempo

Il "quasi" nel titolo di Eco, Dire quasi la stessa cosa, dice della singolarità del traduttore (v. Ponzio 2017), della sua unicità, alterità, della ricerca d'innamorato nell'atto stesso del tradurre, ricerca rispondente alla singolarità del testo, alla sua alterità assoluta, alla sua insostituibilità, di oggetto amato (v. Petrilli 2018).

E proprio per questo il "quasi" di Eco dice della traduzione come presa di posizione, come inevitabile rapporto d'implicazione, di partecipazione responsiva da parte del traduttore nei confronti del testo che traduce, e perciò è indicativo del rapporto dialogico che intercorre sempre tra parola che riporta e parola riportata, malgrado, o forse proprio attraverso, il suo presentarsi sotto la maschera di discorso diretto restituito tale e quale (v. Petrilli 1999: 12; Eco 2003: 20).

La traduzione comporta la responsabilità, quella del traduttore, responsabilità come comprensione rispondente di cui parla Michail Bachtin, una responsabilità responsiva all'unicità, alla singolarità, alla irripetibilità evidenziata da quei testi che rispetto allo stesso, all'identico, all'ovvio, al già dato evidenziano l'insopprimibilità, nell'umano, del "gioco del fantasticare" (Peirce, Sebeok).

Il testo tradotto, per quanto lo stesso resta pur sempre lo "stesso altro" (espressione da me usata occupandomi per caratterizzare il lavoro di traduzione, che Eco in una nota indica come equivalente a quella resa dal suo "quasi" nel titolo da lui scelto per il suo libro sulla traduzione (Petrilli 2001; Eco 2003: 10), non semplicemente perché il testo del traduttore è relativamente altro rispetto all'originale, ma perché il testo originale, testo di scrittura letteraria, resta pur sempre assolutamente altro rispetto al testo che lo traduce. Sicché, come dice Eco (2003: 364), la fedeltà della traduzione consiste nel non dare mai il testo originale come definitivamente tradotto, come testo definitivamente posseduto dal traduttore, ma nel ritenere che la traduzione sia ancora sempre di nuovo possibile. Viceversa, anche qui, come fa Søren Kierkegaard, nel passo citato in epigrafe, a proposito dell'“infedeltà essenziale" nel rapporto amoroso, si può parlare di infedeltà essenziale - cosa abbastanza diffusa dato il ricorrente pretendere, da parte del testo tradotto, il pieno e definitivo possesso del testo originale. 


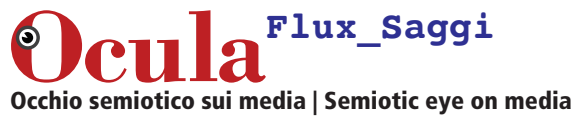

Susan Petrilli | Tradurre il tempo

\section{Bibliografia}

Artuad, Antonin

1961 Il teatro e il suo doppio, pref. di J. Derrida, Torino, Einaudi

1989 L'arve e l'aume, con 24 lettere a M. Baberzat, Parigi, L’Arbalète; tr. it. di L. Feroldi, in Artaud, Il sistema della crudeltà, Millepiani, 11, Milano, Mimesis, pp. 11-19.

Bachtin, Michail M.

1920-24 Per una filosofia dell'azione responsabile, tr. it. di M. De Michiel, introd. di A. Ponzio, Lecce, Manni, 1998.

1979 Estetica slovesnogo tvorčestva, tr. it. di C. Strada Jovanovič, L'autore e l'eroe. Teoria letteraria e scienze umane, Torino, Einaudi, 1988.

Barthes, Roland

1977 Journal de Deuil, 26 octobre 1977 - 15 septembre 1979, texte établi et annoté par Nathalie Léger, Paris, Seuil, 2009; tr. it. di V. Magrelli, Dove lei non è. Diario di lutto, 26 ottobre 1977 - 15 settembre 1979, a cura di Nathalie Léger, Torino, Einaudi, 2010.

1978 Leçon, tr. it. di R. Guidieri, Lezione, Einaudi, Torino 1981, nuova ed., insieme a Sade, Fourier, Loyola (1971), 2001.

1979 Vita nova, in Oeuvres complètes, V, Livres, Textes, Entretiens 1977-1980, Paris, Seuil, 2002.

1980 La chambre claire. Note sur la photographie, Parigi, Seuil; tr. it. di R. Guidieri, La camera chiara. Nota sulla fotografia, introd. di R. Platone, Torino, Einaudi, 1980.

1993-2002 Oeuvres complètes, 5 voll., Parigi, Seuil.

2002a Comment vivre ensemble (1976-77), Parigi, Seuil.

2002b Le Neutre (1977-78), Parigi, Seuil.

2002c "Pierre Loti; 'Aziyadé”" (1972), in Roland Barthes, Euvres Complètes, IV, 1972-1976, Parigi, Seuil.

2003 La préparation du roman I et II. Notes de cours et de séminaires au Collège de France 1978-1979, 1979-1980, a cura di N. Léger, Paris, Seuil; tr. it. La preparazione del romanzo, corsi I e II e seminari al Collège di France (19781979, 1979-1980), tr. it. e cura di J. Ponzio e E. Galiani, Milano, Mimesis, 2010.

2007 Le discours amoureux. Séminaire à l'École pratiques des hautes études 1974-1976, suivi de Fragments d'un discours amoureux (pages inédites), "Les cours et les séminaires de Roland Barthes" sous la direction d'Éric Marty, “Traces Écrites”, Collection dirigée par Dominique Séglard, Paris, Seuil; tr. it. e "Introduzione all'edizione italiana" di Augusto Ponzio, Il discorso amoroso. Seminario a l'École pratique des hautes études 1974-1975, seguito da Frammenti di un discorso amoroso, inediti, Milano, Mimesis, 2015.

Blanchot, Maurice

1955 L'éspace litéraraire, Parigi, Gallimard.

1969 L'entretien infini, Parigi, Gallimard.

Carofiglio, Vito

1966 Nerval e il mito della 'poreté', Firenze, La Nuova Italia.

Eco, Umberto

1984 Semiotica e filosofia del linguaggio, Torino, Einaudi.

1990 I limiti dell'interpretazione, Milano, Bompiani.

1993a La ricerca della lingua perfetta, Roma-Bari, Laterza.

1993b “La traduzione”, in Eco 1993a, pp. 371-379. 


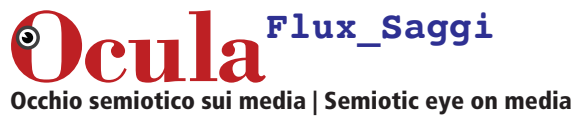

Susan Petrilli | Tradurre il tempo

1994 Sei passeggiate nei boschi narrativi, Milano, Bompiani.

1999a "Experiences in translation", in Giovanna Franci e Siri Nergaard (a cura), La traduzione, numero speciale di Versus. Quaderni di studi semiotici, 82, 87-108.

1999b Traduzione, introduzione e commento a Gerard de Nerval, Sylvie, Torino, Einaudi.

2000 "Traduzione e interpretazione", in Nicola Dusi e Siri Nergaard (a cura), Sulla traduzione intersemiotica, numero speciale di Versus. Quaderni di studi semiotici, 85-87, 55-100.

2001 Experiences in Translation, Toronto, Toronto University Press

2003 Dire quasi la stessa cosa. Esperienze di traduzione, Milano, Bompiani.

Foucault, Michel

1970 L'ordre du discours, tr. it. di A. Fontana, L'ordine del discorso, Torino, Einaudi, 1972.

Jabès, Edmond

1982 La petit livre de la subversion hors de soupçon, Paris, Gallimard; tr. it. di A. Prete, Il libro della sovversione non sospetta, Milano, Feltrinelli, 1984.

Kierkegaard, Søren

1841 Sul concetto di ironia, a c. di D. Bosio, Milano, Guerini, 1989.

1843 Enten-Eller, tr. it. di A. Cortese, 5 voll., Milano, Adelphi, 1976-89.

1845 Stadi del cammino della vita, a c. di L. Koch, Milano, Rizzoli, 1993.

1846 "Postilla non scientifica alle 'Briciole di filosofia”, in Kierkegaard, 1995, vol. 2.

1995 Opere, 3 voll., a cura di C. Fabro, Milano, Piemme.

Levinas, Emmanuel

1974 Autrement qu'etre ou au-delà de l'essence, Nijhoff, Den Haag, $2^{\mathrm{a}}$ ed. 1978; tr. it. di S. Petrosino, M.T. Aiello, introd. di S. Petrosino, Altrimenti che essere o al di là dell'essenza, Milano, Jaca Book, 1983.

1975 Sur Blanchot, Fata Morgana; tr. it. di A. Ponzio, Su Maurice Blanchot, a cura di A. Ponzio e F. Fistetti, Bari, CaratteriMobili.

Loti, Pierre

1971 Aziyadé, Presentazione di Roland Barthes, Milano, Franco Maria Ricci.

Nerval, Gerard de

1887 Sylvie, tr. di Ludvic Halévi, Londra, Routledge.

1932 Sylvie, tr. di Richard Aldigton, Londra, Chatto and Windus.

1965 Les filles du feu. Le chimères, Parigi, Garnier-Flammarion.

1999 Sylvie, nella traduzione di Umberto Eco, Torino, Einaudi.

Peirce, Charles Sanders

2003 Opere, a cura di M. A. Bonfantini, Milano, Bompiani.

Petrilli, Susan (a cura)

1999 La traduzione, nella serie "Athanor. Semiotica, Filosofia, Arte, Letteratura”, X, 2, Roma, Meltemi [ora Milano, Mimesis-Meltemi].

Petrilli, Susan

2000 Tra segni, nella serie "Athanor. Semiotica, Filosofia, Arte, Letteratura”, XI, 3, Roma, Meltemi [ora Milano, Mimesis-Meltemi].

2001 Lo stesso altro, nella serie "Athanor. Semiotica, Filosofia, Arte, Letteratura", XII, 4, Roma, Meltemi [ora Milano, Mimesis-Meltemi].

2009 Signifying and Understanding. Reading the Works of Victoria Welby and the Signific Movement, Berlino, Mouton De Gruyter. 


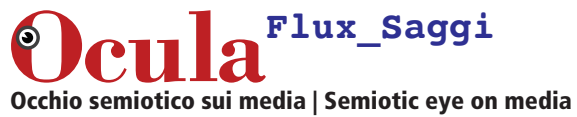

Susan Petrilli | Tradurre il tempo

2010 Sign Crossroads in Global Perspective, Brunswick, Londra, Transactions.

2012a "Tempo di scrittura, tempo di vita nuova”, in Augusto Ponzio et alii, Tempo, corpo, scrittura. PLAT. Quaderni di pratiche linguistiche e analisi di testi, $\mathrm{n}$. 1/2012, 85-112. Lecce, Pensa Multimedia, 2012.

2012b Altrove e altrimenti. Filosofia del linguaggio, critica letteraria e teoria della traduzione in, intorno e a partire da Bachtin, Milano, Mimesis.

2012c Expression and Interpretation in Language, Brunswick, Londra, Transaction.

2013 The Self as a Sign, the World and the Other, Brunswick, Londra, Transaction.

2014a Sign Studies and Semioethics, Berlino, Mouton De Gruyter.

2014b Riflessioni sulla teoria del linguaggio e dei segni, Milano, Mimesis.

2015 Victoria Welby and the Science of Signs, Brunswick, Londra, Transaction.

2016 The Global World and Its Manifold Faces. Transculturalism, Migration, Exploitation, Bern, Berlino, Oxford, New York, Peter Lang.

2017 "La resa del cronotopo letterario di Sylvie di Gérard Nerval nella traduzione di Umberto Eco", Cultura \& Comunicazione. Lingue, Linguaggi Comunicazione / Mass Media / Didattica / Cultura (C\&C), Anno VII, N. 11 - Settembre 2017, 39-42 (doppie colonne), Perugia, Guerra Edizioni, 2017.

2018 "L'immagine dell'altro nel discorso amoroso e nella parola letteraria", in L’immagine nella parola, nella musica e nella pittura, coll. "Athanor. Semiotica, Filosofia, Arte, Letteratura” XXVIII, 21, Milano, Mimesis, 2018, 89130.

Poe, Edgar Allan

1970 Great Tales and Poems, New York, Washington Square Press.

1987 Auguste Dupin, investigatore, Firenze, Passigli.

Ponzio, Augusto

2007 Linguistica generale, scrittura letteraria e traduzione, Perugia, Guerra Edizioni, terza ed., 2012, nuova edizione 2018.

2013 Fuori luogo. L'esorbitante nella riproduzione dell'identico, Milano, Mimesis.

2016 La coda dell'occhio. Letture del linguaggio letterario senza confini nazionali, Roma, Arcne.

2017 "Il 'quasi' del dire quasi la stessa cosa come responsabilità senz'alibi del traduttore", Cultura \& Comunicazione. Lingue, Linguaggi Comunicazione / Mass Media / Didattica / Cultura (C\&C), Anno VII, 11, Settembre 2017, 4348, Perugia, Guerra Edizioni, 2017.

Ponzio, Augusto; Petrilli, Susan,

1999 Fuori campo. I segni del corpo tra rappresentazione ed eccedenza, Milano, Mimesis.

2003 Views in Literary Semiotics, Ottawa, New York, Legas.

2005 Semiotics Unbounded. Interpretive Routes in the Open Network of Signs, Toronto, Toronto University Press.

2006 La raffigurazione letteraria, Milano, Mimesis.

2016 Lineamenti di semiotica e di filosofia del linguaggio. Un contributo all'interpretazione del segno e all'ascolto della parola, Perugia, Guerra Edizioni.

Proust, Marcel

1954 Contre Sainte-Beuve suivi de Nouveaux mélanges, Paris, Gallimard.

1974 Contro Sainte-Beuve, tr. it. di P. Serini e M. Bertini dall'edizione critica a cura di Pierre Clarac, saggio introduttivo di F. Orlando, Torino, Einaudi (i riferimenti dei passi in italiano nel testo riguardano questa traduzione).

2008 Alla ricerca del tempo perduto, tr. di M. Bongiovanni Bertini, Torino, Einaudi. 2013 Contro Sainte-Beuve, note al testo di Pierre Clarac, Milano, Mimesis. 


\section{Dcula ${ }^{\text {Flux__aggi }}$ \\ Occhio semiotico sui media | Semiotic eye on media}

Susan Petrilli | Tradurre il tempo

Queneau, Raymond

1983 Esercizi di stile, tr. di Umberto Eco, Torino, Einaudi.

Sebeok, Thomas A.

1981 The Play of Musement, Bloomington, Indiana University Press; tr. it. di M. Pesaresi, Il gioco del fantasticare, Milano Spirali, 1984.

Violi, Patrizia (a cura)

1982 Versus. Quaderni di studi semiotici, 31/32, numero speciale, Su Sylvie (con contributi di Patrizia Violi, Isabella Pezzini, Beppe Cattafavi, Daniele Barbieri, Mia Pia Pozzato, studenti e ricercatori che hanno partecipato al seminario del 1976-1977, tenuto presso la cattedra di Semiotica nell'Istituto di Comunicazioni, Università di Bologna). In appendice, Gérard Nerval, Sylvie.

Wittgenstein, Ludwig

1922 Tractatus logico-philosophicus, tr. it. di A. G. Conte, in Tractatus logicophilosophicus e Quaderni 1914-16, Torino, Einaudi, 1980.

Susan Petrilli, professore di Filosofia e teoria dei linguaggi, insegna Semiotica e Semiotica della traduzione nell'Università di Bari Aldo Moro. È Visiting Research Fellow nella Adelaide University, Australia. Fra le sue pubblicazioni: Signifying and Understanding. Reading the Works of Victoria Welby and the Signific Movement, Mouton, 2009; The Self as a Sign, the World and the Other, Transaction, 2013; Sign Studies and Semioethics, Mouton, 2014; The Global World and Its Manifold Faces, Peter Lang, 2016; con Augusto Ponzio Lineamenti di semiotica e di filosofia del linguaggio, Guerra Ed., 2016; L’immagine nella parola, nella musica e nella pittura (a cura), Mimesis, 2018. 